\title{
Leitthema
}

Gefässchirurgie 2018 $23: 420-428$ https://doi.org/10.1007/s00772-018-0442-4 Online publiziert: 17. September 2018 (c) Der/die Autor(en) 2018

CrossMark

\author{
M. K. Widmer $\cdot$ A. Arnold ${ }^{2}$ D. E. Uehlinger ${ }^{3} \cdot$ U. Dietrich $^{3} \cdot$ M. Caversaccio $^{2}$ \\ ' Universitätsklinik für Herz-und Gefässchirurgie, Inselspital, Universität Bern, Bern, Schweiz \\ ${ }^{2}$ Universitätsklinik für Hals-, Nasen- und Ohrenheilkunde, Inselspital, Universität Bern, Bern, Schweiz \\ ${ }^{3}$ Universitätsklinik für Nephrologie und Hypertonie, Inselspital, Universität Bern, Bern, Schweiz
}

\section{Retroaurikulär fixierter Port zur Hämodialyse: erste klinische Erfahrung}

\section{Einführung}

Für Hämodialyse(HD)-Patienten ist ein funktionierender Gefäßzugang überlebenswichtig. $\mathrm{Zu}$ feine oder arteriosklerotisch veränderte Arterien respektive schmale und vernarbte Venen oder eine schwere kardiale Belastung verunmöglichen es nicht selten, bei dieser immer älteren und polymorbideren Patientenpopulation eine arteriovenöse (AV-) Fistel resp. einen Kunststoffshunt anzulegen. Für diese Patienten bleibt neben der Option einer Peritonealdialyse nur die Einlage eines subkutan tunnelierten HD-Katheters.

Katheter können verstopfen und im Langzeitverlauf $\mathrm{zu}$ zentralen Venenthrombosen führen. Verschiedene Studien haben zudem gezeigt, dass bei Kathetern die Infektrate deutlich erhöht und dies wiederum mit einer höheren Mortalität vergesellschaftet ist [3, 9, 10]. Die Hazard Ratio für infektiöse Ereignisse bei permanenten tunnelierten Kathetern im Vergleich zu AV-Fisteln beträgt 1,95 (95\%-Konfidenzintervall [-KI] 1,47-2,57; [7]).

》) Infekt- und Mortalitätsrisiko bei Kathetern höher als für AV-Grafts und am geringsten für AV-Fisteln

Eine Metaanalyse von 2016 bestätigt eine allseits bekannte Tatsache, dass AVFisteln bezüglich primärer Offenheit mit $55 \%$ nach 2 Jahren deutlich besser sind als für AV-Grafts mit $40 \%$ und für Katheter mit $50 \%$. Das Infekt- und Mortalitätsrisiko ist bei Patienten mit Kathetern höher als für AV-Grafts und am geringsten für AV-Fisteln [1]. Auch die Raten für Bakteriämien permanenter Katheter sind mit 4,2 Ereignissen auf 100 Patientenmonate, resp. 27,1 für nicht tunnelierte Katheter, deutlich höher als für AV-Fisteln mit 0,5 resp. AV-Grafts mit 0,9 [8].

In der Hals-Nasen-Ohrenheilkunde (HNO) werden seit Jahren retroaurikulär fixierte Implantate zur Verbesserung der Hörfähigkeit eingebaut. Diese Systeme haben eine erstaunlich niedrige lokale

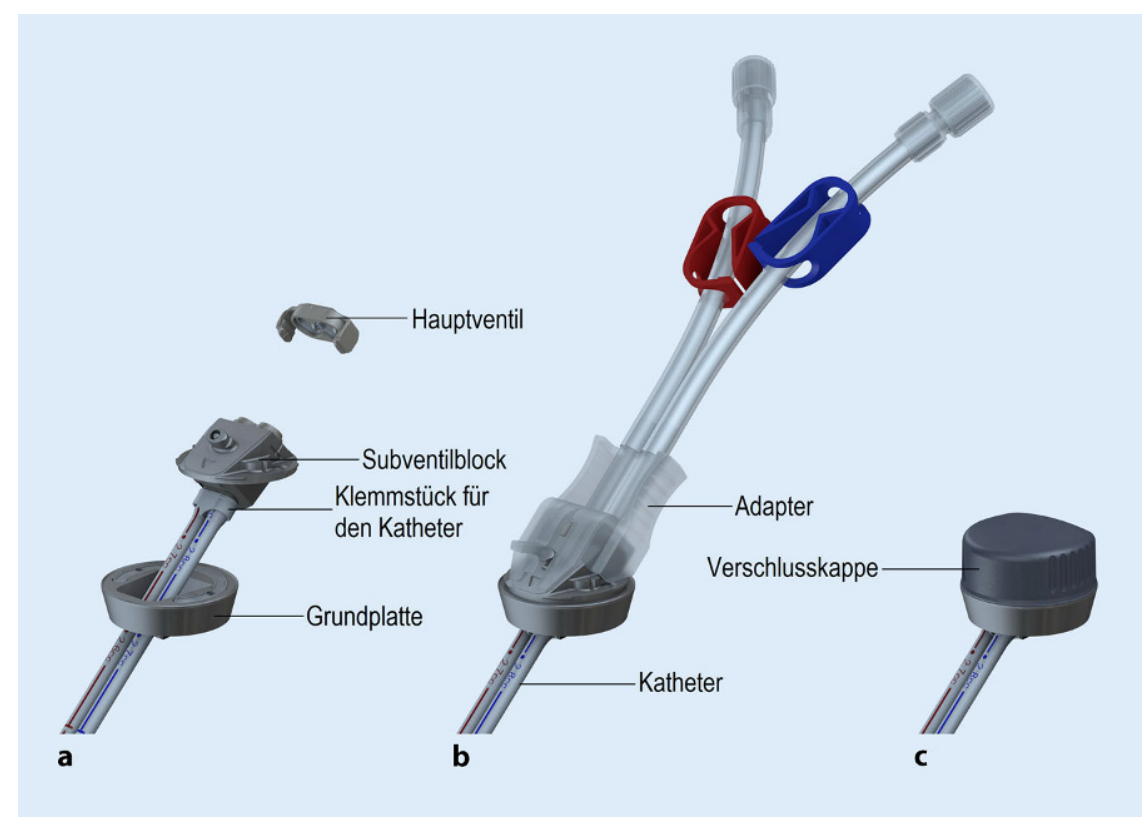

Abb. 1 A Systemkomponenten des Ports: a Fixation des HD-Katheters am Subventilblock mit dem Hauptventil, das alle 4 Wochen gewechselt werden muss. b Aufgesetzter Adapter zur Konnektion mit den Schläuchen der Dialysemaschine. c Port mit Schutzkappe. (Mit freundl. Genehmigung von Diavantis, dieser Inhalt ist nicht Teil der Open-Access-Lizenz) 
mit der Industrie (Fa. Cendres + Métaux) zusammen einen auf dem Mastoidknochen verankerten Port („bone-anchored port“, BAP) zu entwickeln, welcher mit einem herkömmlichen tunnelierten HDKatheter verbunden wird [12]. Während der Entwicklungsphase wurden hämodynamische Studien durchgeführt, um genügende Flussvolumina $\mathrm{zu}$ erreichen [6].

Im Folgenden werden die Operationstechnik und erste klinische Resultate präsentiert. Die Studie wurde von der kantonalen Ethikkommission Bern und Swissmedic bewilligt. Aktuell können Patienten in die Studie eingeschlossen werden, bei denen man zur Schaffung eines HDZugangs Fremdmaterial benötigt.

\section{Methode}

\section{Das System}

Zuerst wird mit Hilfe von dafür entwickelten Schablonen eine Vertiefung in den Knochen und eine ossäre Rinne Richtung Mastoidspitze/Kieferwinkel gefräst [12]. Danach wird das Portsystem, bestehend aus einer Grundplatte aus Reintitan von $29 \mathrm{~mm}$ Durchmesser, mit 8 Schrauben auf den Mastoid fixiert. Jetzt wird vom Hals her der Katheter retrograd subkutan und danach durch die mastoidale Rinne und die Grundplatte durchgezogen und mit dem Innengehäuse fixiert. Das Innengehäuse enthält einen Subventilblock, welcher das Herzstück des Ports bildet. Es wird anschließend auf die Grundplatte geschraubt (• Abb. 1).

Ein spezieller Adapter erlaubt es, mit einem Handgriff den Port mit den Blutschläuchen des Dialysegeräts zu konnektieren. Nach Abschluss der Dialyse wird der Adapter entfernt und das System wieder mit einer Verschlusskappe geschützt. Die Hauptventilkomponente, ein Silikonschlitzventil, muss durch das Pflegefachpersonal alle 4 Wochen oder nach 12 Dialysen gewechselt werden. Dies lässt sich durch Betätigung einer Klemmbacke im Subventil, zur Verhinderung einer Luftembolie und einem Demontage- und einem Montagewerkzeug mit einem bereits vormontierten neuen Hauptventil einfach bewerkstelligen.

\section{Präoperative Abklärung}

Zur Indikationsstellung werden die V. juglaris interna beidseits resp. die V. cava superior mittels Duplex auf ihre Offenheit untersucht. Sind diese Voraussetzungen gegeben und die $\mathrm{Pa}$ tienteneinwilligung eingeholt, wird im nächsten Schritt eine Computertomographie (CT) des Schädels durchgeführt, um die retroaurikuläre Knochendicke festzustellen. Diese sollte $6 \mathrm{~mm}$ betragen, damit die notwendige Vertiefung gefräst und die Knochenschrauben zur Fixation der Grundplatte ohne Verletzung der Dura eingebracht werden können. Hierzu wird aus den CT-Daten eine Knochendickekarte erstellt ([5]; - Abb. 2).

\section{Implantationstechnik}

Bei dem in Vollnarkose liegenden Patienten wird nach Rasur und Desinfektion entsprechend der CT-Untersuchung die optimale Lage des Systems im Vergleich zum Gehörgang markiert und die Zirkumferenz des Ports markiert. Nach Mobilisation des Hautlappens wird der Mas- toidknochen freigelegt, das Periost lokal entfernt und mit einer ersten Schablone zwei Pilotbohrungen durchgeführt. Diese Bohrlöcher erlauben es, alle späteren für die Knochenbearbeitung notwendigen Schablonen immer identisch zu positionieren (• Abb. 3). Mit einem Ringhobel (Diamantfräse) wird die zirkuläre Kontur der Grundplatte geschaffen. Unter Verwendung von drei weiteren Schablonen werden die Vorbohrungen für die Knochenschrauben und die Gehäusekavität gemacht. Danach kann der mastoidale Kanal für die Verlegung des HDKatheters und die Knochentunnelierung präpariert werden (•Abb. 4, 5, 6 und 7). Die Vertiefungen werden wahlweise mit einem Rosen- oder Diamantbohrer gefräst. Dabei ist insbesondere bei der Präparierung der Gehäusekavität und der Knochentunnelierung auf eine Schonung der Dura oder des Sinus sigmoideus zu achten.

\section{Katheterplatzierung}

Passt die Grundplatte mit dem Subventilblock gut in die vorbereitete Knochenvertiefung wird nun ca. 2 Querfinger oberhalb des Schlüsselbeins über dem M. sternocleidomastoideus mit dem Ultraschall die V. jugularis interna identifiziert. Es erfolgt eine kleine Hautinzision und es wird ein feiner subkutaner Tunnel geschaffen. In Trendelenburg-Lage wird unter Ultraschallkontrolle die V. jugularis interna punktiert und ein Führungsdraht eingebracht. Damit wird die benötigte Katheterlänge abgemessen, deren Spitze unter Bildwandlerkontrolle am Übergang von der V. cava superior in den rechten Vorhof lokalisiert wird. Der venöse Zugang wird nun schrittweise aufdilatiert und

Hier steht eine Anzeige. 


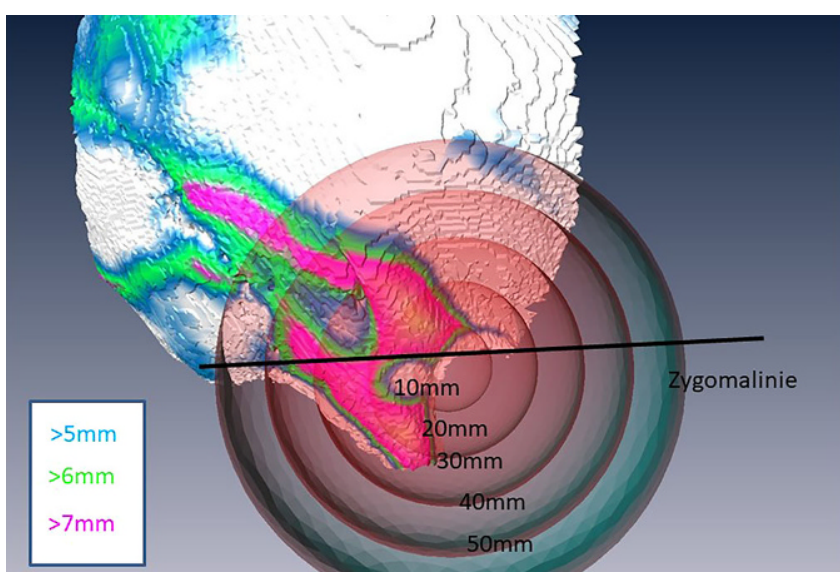

Abb. $2 \Delta$ CT-Planungsaufnahme, damit die Zone mit der größten Knochendicke (gefordert sind $>6 \mathrm{~mm}$ ) zur optimalen Platzierung der Grundplatte bestimmt werden kann. (Mit freundl. Genehmigung Inselspital Bern, dieser Inhalt ist nicht Teil der Open-Access-Lizenz)

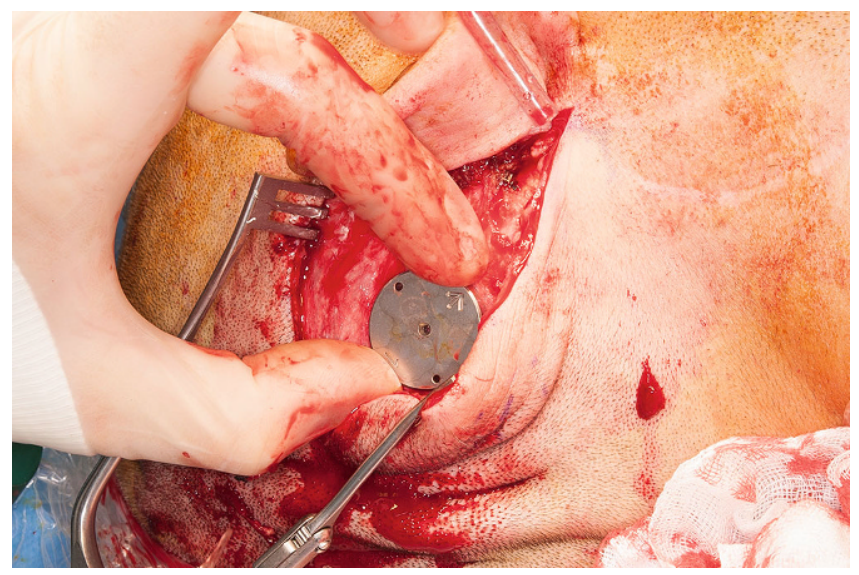

Abb. 3 ॥ Nach Markierung der genauen Position des Ports auf dem Mastoid wird ein Hautlappen gebildet, das Periost an der markierten Stelle entfernt und mit einer Schablone die Position des Ports durch Anbringen von zwei feinen Bohrlöchern markiert. (Mit freundl. Genehmigung, @Prof. Marco Caversaccio und Gianni Pauciello. Alle Rechte vorbehalten, dieser Inhalt ist nicht Teil der Open-Access-Lizenz) am Schluss die Splitschleuse eingebracht. Über diese kann nun antegrad der mit Heparin-Lösung gefüllte Katheter eingebracht werden. Unter Bildverstärkung wird die Katheterspitze ideal platziert und danach der Katheter retrograd im Halsbereich und durch den vorgefertigten Knochenkanal und die Grundplatte durchgezogen (• Abb. 8). Nach Montage von provisorischen Katheteranschlüssen erfolgt ein Aspirations- und Instillationstest des Katheters. Bei guter Lage und Funktionstüchtigkeit wird der Katheter auf die korrekte Länge gekürzt.

\section{Abschluss der Operation}

Nun wird der Katheter mit dem Subventil verbunden und dieses in die Grundplatte geschoben und dort mit zwei Schauben fixiert. Nach einer finalen Lagekontrolle der Katheterspitze wird mit dem fixierten Subventil nach Aufsetzen des Adapters das gesamte Portsystem nochmals mit dem Aspirations- und Instillationstest geprüft (• Abb.9). Das Kathetersystem wird zuletzt mit Taurolin/Heparin-Lock-Lösung gefüllt. Zum Abschluss wird im Hautlappen ein kleines Loch von $2 \mathrm{~cm}$ Durchmesser ausgeschnitten, subkutan sparsam ausgedünnt und der Hautlappen satt über das Portsystem gestülpt, um eine gute seitliche Abdichtung zu erzielen ( Abb. 10). Nach Desinfektion wird die Halsinzision und die retroaurikuläre Inzision mittels Naht verschlossen.

\section{Ergebnisse}

Von April 2016 bis April 2018 wurden 3 Männer und eine Frau in diese PhaseI/II-Studie eingeschlossen (•Tab. 1). Initial konnte lange kein Patient eingeschlossen werden, weil das ursprüngliche Studienprotokoll als Einschlusskriterium nur die Alternative zu einem permanenten Katheter vorsah. Der Entscheid zu einem permanenten Katheter erfolgt allerdings in den meisten Fällen sehr kurzfristig und nur, wenn keine andere Alternative mehr vorliegt. Dieses Vorgehen ist nicht mit dem Planungsaufwand für das Anlegen eines BAP vereinbar. Erst als die Ethikkommission des Kantons Bern und die Zulassungsbehörde Swissmedic grünes Licht für eine Erweiterung der Einschlusskriterien gaben, konnten Patienten für die Studie rekrutiert werden. Die aktuellen Einschlusskriterien sehen vor, dass ein BAP angelegt werden kann, wenn keine Möglichkeit eines Gefäßzugangs ohne Fremdmaterial besteht.

Das Durchschnittsalter der 4 Patienten betrug 60 (Range 36-83) Jahre. Intraoperativ musste einmal ein Liquorleck durch ein Bohrloch mit Knochenwachs versiegelt werden. Bei einem Patienten war das Hautloch zu groß ausgeschnit- ten, so dass man im frühen postoperativen Verlauf diese Wunde plastisch verkleinern musste. Im Schnitt wurde das System 9 (Range 2-15) Tage nach Anlage erstmals verwendet. Bisher wurde kumulativ 985 Tage dialysiert.

Der erste Patient wurde über ein Jahr problemlos über das Portsystem dialysiert. Bei diesem Patienten musste früh postoperativ das Subventil wegen einer verklebten Klemmbacke ausgetauscht und nach 13 Monaten nochmals gewechselt werden, weil die Klemmbacke nach einem Hauptventilwechsel leicht obstruierte. Letzteres führte $\mathrm{zu}$ einer technischen Änderung an der Klemmbacke des Subventils. Beide Wechsel erfolgten ambulant und ohne jegliche Anästhesie. Der Katheter ließ sich für beide Subventilwechsel widerstandslos ein Stück herausziehen und wieder hineinschieben. Leider verstarb der erste Patient 14 Monate nach Implantation an einer schweren Herzinsuffizienz in Kombination mit einer diabetesbedingten Hypoglykämie. Keiner der Patienten musste bisher wegen eines Katheterinfekts behandelt werden. In 2 Fällen kam es zu einer Abnahme der Blutflusskapazität, welche mit Urokinase-Infusionen problemlos behandelt werden konnten. Bisher musste kein Katheter wegen einer Thrombosierung oder eines Infekts ausgewechselt werden. 


\section{Erste Dialyseerfahrungen}

Die Pflege des Ports und die Konnektion vor und die Dekonnektion nach der Dialyse sind einfach und für den Patienten völlig schmerzfrei. Das Aufsetzen des Adapters und das Anbringen des Deckels nach Dialyseende muss geübt werden. Die Dialyse kann problemlos mit einem Fluss von $350 \mathrm{ml} / \mathrm{min}$ gefahren werden, wobei sich die arteriellen und venösen Drücke im Normbereich bewegen. Bei allen Patienten, außer Patient Nr. 2, zeigten rasch reizlose, trockene Wundverhältnisse. Krusten können problemlos mit $\mathrm{NaCl}$ $0,9 \%$ gelöst werden. Der Port darf unmittelbar nach Implantation für die Dialyse benutzt werden, dies ist jedoch durch die frühpostoperative Schwellung und Empfindlichkeit des Gewebes um den Port für den Patienten schmerzhaft. Bei $2 \mathrm{~Pa}$ tienten kam es im Verlauf zur Bildung von Hypergranulationsgewebe um den Port, welche das Montieren des Adapters resp. das Aufbringen der Schutzkappe erschweren. Ein nicht erwartetes Problem sind zudem nachwachsende Haare, welche die Manipulation am Port erschweren, weil man vermeiden muss, dass sich Haare im System verfangen.

\section{) Die Pflege des Ports und die Konnektion vor und die Dekonnektion nach der Dialyse sind einfach}

Alle Patienten sind zufrieden mit dem System. Anfängliche Bedenken in Bezug auf Schmerzen, Ohrgeräuschen, Schlafstörungen oder Auffälligkeiten im Aussehen durch den Port haben sich nicht bestätigt. Patienten, welche den Port mit anderen Dialysezugängen vergleichen können, empfinden diesen als sicherer und weniger belastend. Im Alltag wird der Port von den Patienten nicht mehr wahrgenommen, das Schlafen auf der Portseite ist nach dem initialen Heilungsprozess problemlos möglich und, sobald die Haare nachgewachsen sind.

Gefässchirurgie 2018 $23: 420-428$ https://doi.org/10.1007/s00772-018-0442-4

(c) Der/die Autor(en) 2018

M. K. Widmer · A. Arnold · D. E. Uehlinger - U. Dietrich · M. Caversaccio

\section{Retroaurikulär fixierter Port zur Hämodialyse: erste klinische Erfahrung}

\section{Zusammenfassung}

Hintergrund. Retroaurikulär fixierte Hörhilfen sind in der Regel wenig anfällig für Infekte im Gegensatz zu Kathetern für die Hämodialyse (HD), welche transkutan/subkutan eingelegt werden. Diese weisen häufig Infekte als Komplikation mit der dazugehörigen Morbidität und Mortalität auf. Ein neu entwickelter retroaurikulär am Knochen fixierter Port in Kombination mit einem konventionellen tunnelierten HD-Katheter wird aktuell bezüglich Sicherheit und Funktionalität für die HD getestet. Methode. Im April 2016 wurde der seit 2010 von Ärzten und Industrie entwickelte retroaurikulär fixierte Port (,,bone-anchored port", BAP) erstmals einem Menschen implantiert. In dieser Phase-I/II-Studie geht es darum, die entworfenen Standards zur Implantation dieses Systems zu überprüfen und ggf. anzupassen, aber auch deren Handhabung und die Dialysequalität mit prospektiven Daten zu erfassen.

Ergebnisse. Bisher konnten 4 Patienten über diesen Zugang durchschnittlich ab dem 9. Tag nach Implantation (Range 2-15) problemlos dialysiert werden. Bis dato haben wir 985 (Ø 246, Range 138-445) Patiententage erreicht. Ein Patient ist unabhängig vom System 14 Monate nach der Implantation verstorben. Probleme mit dem Subventil führten beim ersten Patienten zu einer Anpassung des Systems.

Schlussfolgerungen. Die Implantation eines retroaurikulär fixierten Ports braucht ein interdisziplinäres Team, kann aber mit der nötigen Sicherheit durchgeführt werden und wird von den Patienten und Pflegenden wegen der einfachen Handhabung sehr geschätzt. Es braucht eine gute Schulung des chirurgischen Teams und des Pflegefachpersonal, damit sich diese Methode weiter etablieren kann und mehr Daten zur Funktionalität und Performance dieses Systems erfasst werden können.

Schlüsselwörter

Chronische Niereninsuffizienz · Shunt · Port . Katheter - Cochlea-Implantat

\section{Bone-anchored port for hemodialysis: preliminary clinical experience}

\section{Abstract}

Background. Bone-anchored hearing aids seem to be more resistant to infections compared with non-/tunneled catheters for hemodialysis. Catheter-related bloodstream infections are associated with morbidity and mortality. A newly designed bone-anchored port (BAP) connected to a conventional hemodialysis catheter is currently under evaluation for safety and functionality. Methods. Starting in 2010, physicians and engineers began developing this BAP which was implanted for the first time in a human in April 2016. The aim of this pilot study is to evaluate the implantation procedure, but also with a prospective follow-up to obtain experience with handling of the device during dialysis and to observe the quality of dialyses. Results. Until April 1, 2018 the BAP was implanted into 4 patients. Currently 985 patient days (average 246 days, range 138-445 days) have been achieved. One patient died unrelated to the device after 14 months. A problem with the inner valve resulted in a technical change after the first patient.

Conclusions. An interdisciplinary team is needed to implant the device, which can be implanted in a safe manner and is appreciated by patients and nurses due to its simple handling. An intensive education program is mandatory in order to establish this method and to obtain more data concerning functionality and performance.

Keywords

End stage renal disease - Shunt - Catheter . Port - Cochlear implant 


\section{Leitthema}

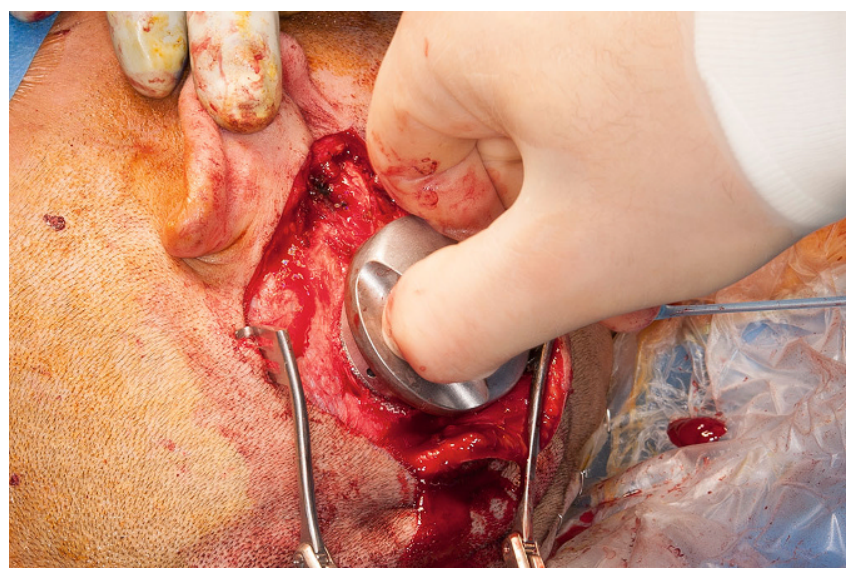

Abb. 4 \ Mit einer Diamantfräse wird die Kontur zur Verankerung der Grundplatte geschaffen. (Mit freundl. Genehmigung, @Prof. Marco Caversaccio und Gianni Pauciello. Alle Rechte vorbehalten, dieser Inhalt ist nicht Teil der Open-Access-Lizenz)

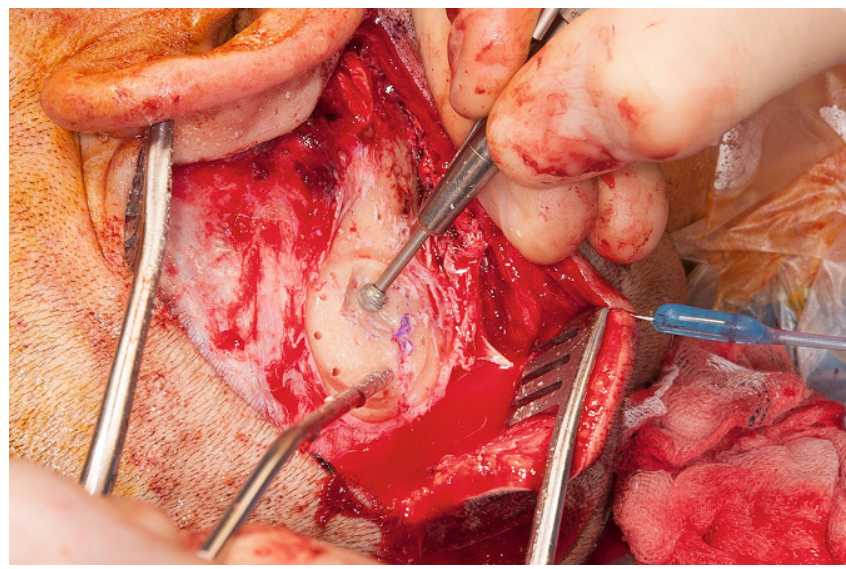

Abb. $6 \Delta$ Nach Setzen der Bohrlöcher wird mit einem Diamantbohrer die Kavität für den Kathetereinlass in die Grundplatte ausgefräst. (Mit freundl. Genehmigung, @Prof. Marco Caversaccio und Gianni Pauciello. Alle Rechte vorbehalten, dieser Inhalt ist nicht Teil der Open-Access-Lizenz)

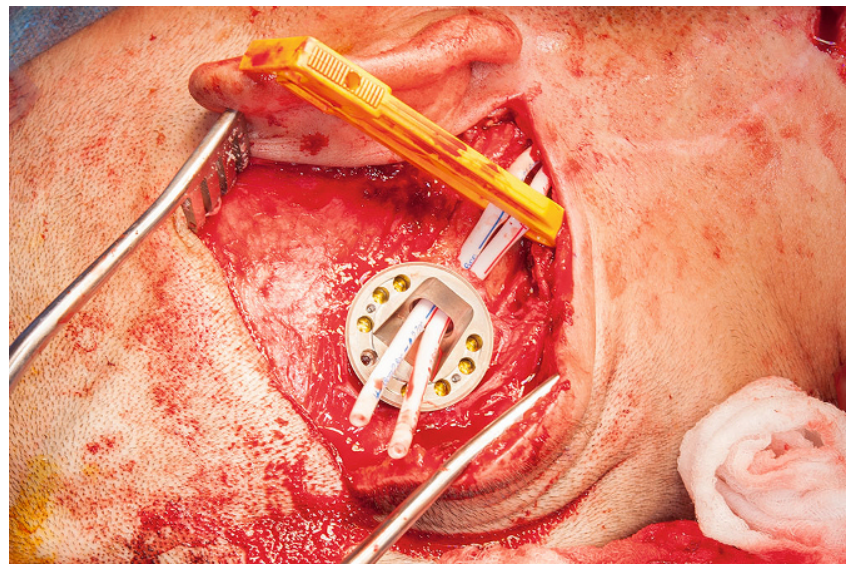

Abb. 8 ॥ Nach erfolgreicher Einlage des Katheters über die V.jugularis interna vor den rechten Vorhof wird dieser retrograd subkutan durchgezogen und danach durch den Knochenkanal und Grundplatte ausgeleitet. (Mit freundl. Genehmigung, OProf. Marco Caversaccio und Gianni Pauciello. Alle Rechte vorbehalten, dieser Inhalt ist nicht Teil der Open-Access-Lizenz)

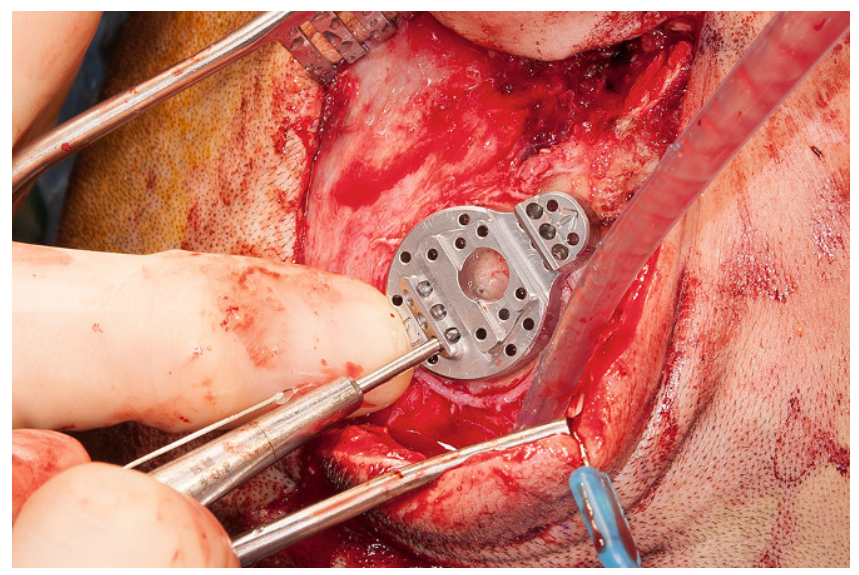

Abb. 5 A Unter Benutzung einer zweiten Schablone werden die Bohrlöcher für die Grundplatte gesetzt. (Mitfreundl.Genehmigung, @Prof. MarcoCaversaccio und Gianni Pauciello. Alle Rechte vorbehalten, dieser Inhalt ist nicht Teil der Open-Access-Lizenz)

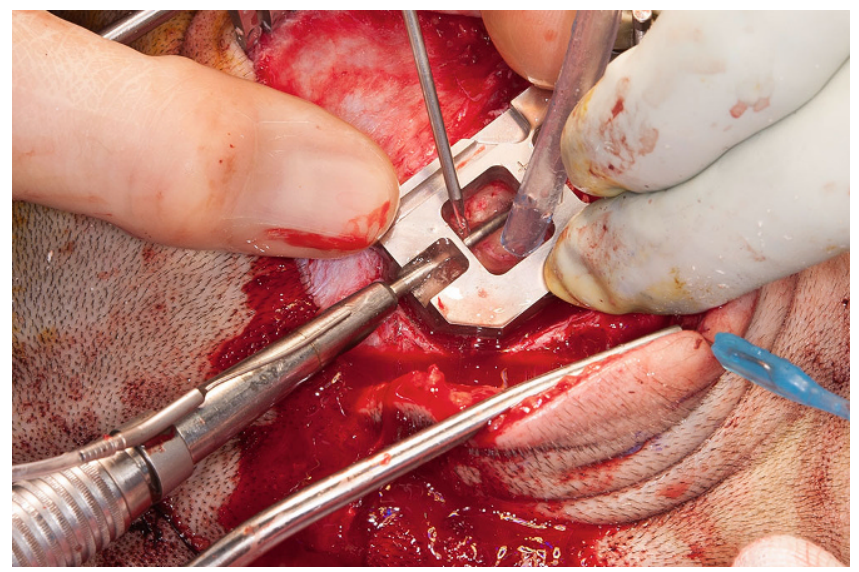

Abb. 7 ॥ Eine Schablone und ein Bohrer mit einer fixierten Länge erlauben die präzise Bohrung des ossären Katheterdurchzugskanals. (Mit freundl. Genehmigung, @Prof. Marco Caversaccio und Gianni Pauciello. Alle Rechte vorbehalten, dieser Inhalt ist nicht Teil der Open-Access-Lizenz) 


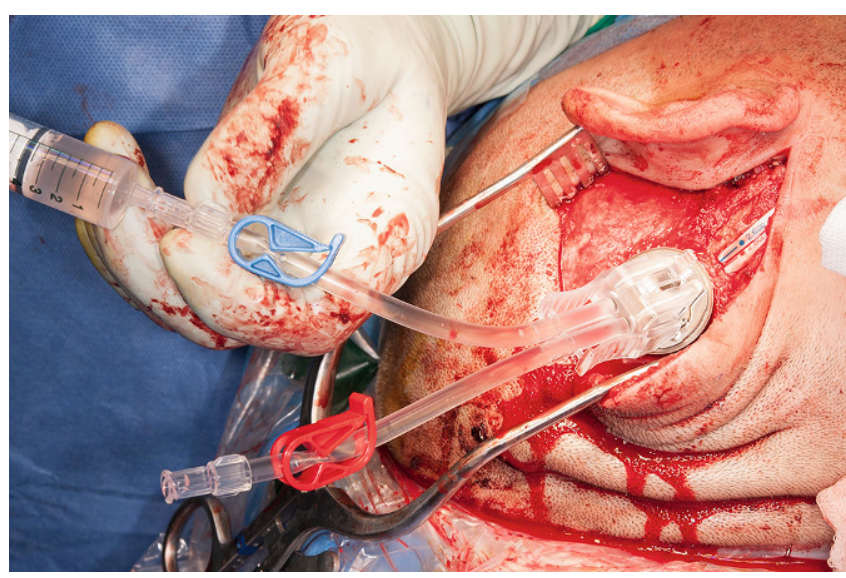

Abb. 9 ॥ Testen der korrekten Katheterfunktion unter Verwendung eines Adapters nachdem man die Katheterspitze korrekt platziert und den Katheter an den Subventilblockfixiert hat. (Mitfreundl.Genehmigung, $\odot$ Prof.Marco Caversaccio und Gianni Pauciello. Alle Rechte vorbehalten, dieser Inhalt ist nicht Teil der Open-Access-Lizenz)

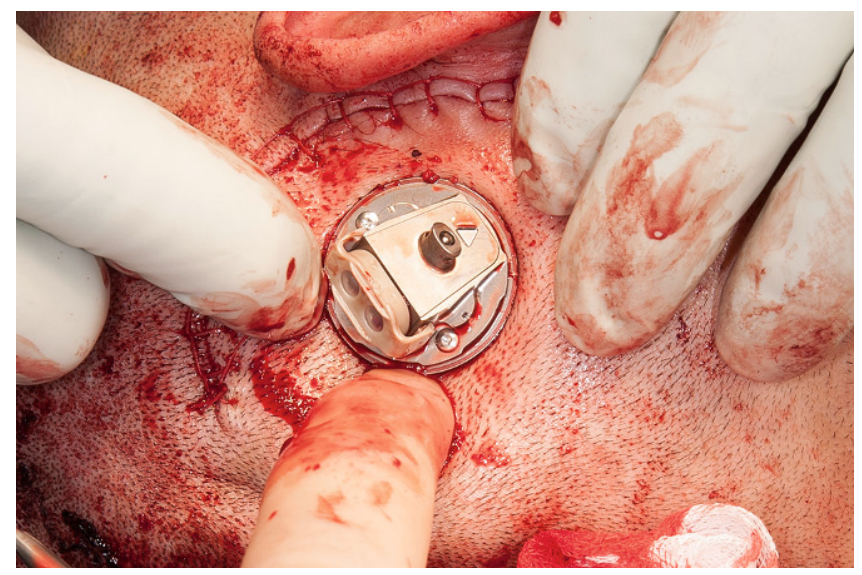

Abb. $10 \Delta$ Einpassen der Haut um die Grundplatte mit aufgeschraubtem Subventil nachdem man den Hautlappen wieder zurück verlagert hat. (Mit freundl. Genehmigung, $\odot$ Prof. Marco Caversaccio und Gianni Pauciello. Alle Rechte vorbehalten, dieser Inhalt ist nicht Teil der Open-Access-Lizenz) verschiedenen Schablonen als Hilfe zur Implantation verinnerlichen.

Vom Gefäßchirurgen oder Interventionalisten wird verlangt, dass er die Handgriffe der Kathetereinlage mit der nötigen Sicherheit beherrscht und im Vorfeld abschätzen kann, ob eine Kanülierung erfolgreich sein wird. Nicht zuletzt ist die Pflegeassistenz im Operationssaal entscheidend, damit die Arbeitsabläufe unter Verwendung von Ultraschall, Bildverstärker und verschiedensten Instrumenten und technischen Hilfsmitteln zeitverzugslos funktioniert. Teams, welche dieses System implantieren wollen, werden eine Lernkurve haben. Es ist zwingend, dass die einzelnen Operationsschritte klar definiert sind. Computergestützte Operationsanleitungen sind in Vorbereitung, um den Implantationsalgorithmus im Operationssaal sicherzustellen.

Obwohl erst 4 Patienten in diese Phase-I/II-Studie eingeschlossen werden konnten, fällt die bisherige Bilanz positiv aus. Bei allen 4 Patienten war eine Implantation technisch möglich. Die präoperativ durchgeführte Messung der Schädeldicke ist essentiell. Es ist wichtig, bei konkavem Knochen diesen auszugleichen, damit die Grundplatte gut aufliegend fixiert werden kann. Die verschiedenen Schablonen zur Konfiguration der Vertiefung für das Subventil respektive des Kanals mit Schaffung ei- ner Knochenbrücke zum Durchzug des Katheters haben sich bewährt.

\section{) Es ist zwingend, dass die einzelnen Operationsschritte klar definiert sind}

Blutungen aus dem Knochen können mit Hitze durch einen nicht mit Wasser gekühlten Diamantbohrer gestillt werden. Einmalig hat sich bei einem Bohrloch etwas Liquor entleert. Zur Abdichtung wurde Knochenwachs verwendet. Alternativ kann Knochenmehl mit Fibrin aufgebracht werden, um den Defekt abzudichten.

Die Venenpunktion unter Ultraschall zur Einlage des Katheters hat bisher gut funktioniert. Mit einem normierten Führungsdraht lässt sich unter dem Bildverstärker die Länge bestimmen, damit man die korrekte Katheterlänge wählt. Man muss sich bewusst sein, dass der Katheter temporär durch die Grundplatte etwas hochgezogen werden muss, damit man das Ventil sicher und fest anschließen kann. Manipulationen am Katheter sollen immer in Trendelenburg-Lage erfolgen, und es muss peinlich darauf geachtet werden, dass die beiden Katheterlumen mit einer weichen Klemme blockiert sind. Es hat es sich bewährt, nach Aufbringen des Adapters mit einer Spritze durch rasche Aspiration und Instillation zu testen, ob ein genügendes Blutvolumen gefördert werden kann.

Im postoperativen Verlauf sind lokale Wundprobleme im Bereich des Ports eine Herausforderung. Bei einem Patienten war eine lokale plastische Deckung notwendig, um ein zu groß ausgeschnittenes Loch um den Port zu verkleinern. Im Verlauf konnte bei diesem Diabetiker mit einer anamnestisch ausgeprägten Neigung zu Infekten und Wundheilungsstörungen ein lokaler Hautinfekt um den Port mit Antibiotika und Antiseptikagazen erfolgreich behandelt werden.

Eine sorgfältige und regelmäßige Pflege der Wunden ist unabdingbar, auch weil es gerne zu Hypergranulation an der Grenzzone zum Port kommen kann. Erfreulich ist, dass es bei kumulativ über 900 Dialysetagen bei allen 4 Patienten bisher zu keiner Katheterinfektion gekommen ist, und dass auch keine Katheterthrombosen registriert werden mussten.

Die lange Phase der technischen Entwicklung dieses Ports hat sich bisher gelohnt, funktioniert doch der Port während der Dialyse ausgezeichnet. Die geforderte Flussmenge von $>300 \mathrm{ml}$ Fluss pro Minute wird während den Dialysen problemlos erreicht und die Qualität der Dialyse stimmt. Die einfache Handhabung des Adapters zum Anschluss an die Dialysemaschine ist für die Arbeit der Pflegenden eine Erleichterung. Der Schließmechanismus (Klemmbacke) im 


\begin{tabular}{|c|c|c|c|c|c|}
\hline Patient & Geschlecht & $\begin{array}{l}\text { Alter } \\
\text { (Jahre) }\end{array}$ & Grunderkrankung & Nebendiagnosen & $\begin{array}{l}\text { Besonderes/Indikation } \\
\text { zum Einbau }\end{array}$ \\
\hline \multirow[t]{3}{*}{1} & \multirow[t]{3}{*}{ Männlich } & \multirow[t]{3}{*}{81} & \multirow[t]{3}{*}{$\begin{array}{l}\text { Multifaktorielle Niereninsuffizienz bei } \\
\text { monoklonaler Gammopathie }\end{array}$} & $\begin{array}{l}\text { Rezessive Pankreatitiden, } \\
\text { St. } n \text {. Whipple-Operation } \\
\text { bei Pankreaskarzinom, se- } \\
\text { kundärer Diabetes mellitus }\end{array}$ & $\begin{array}{l}\text { Schlechte Venenqualität für } \\
\text { eine Shuntanlage }\end{array}$ \\
\hline & & & & $\begin{array}{l}\text { Verdacht auf multilokuläres } \\
\text { Nierenzellkarzinom bds. }\end{array}$ & Kardiale Situation \\
\hline & & & & $\begin{array}{l}\text { Schwere hypertrophe und } \\
\text { valvuläre Kardiopathie }\end{array}$ & - \\
\hline \multirow[t]{5}{*}{2} & \multirow[t]{5}{*}{ Männlich } & \multirow[t]{5}{*}{63} & \multirow[t]{5}{*}{$\begin{array}{l}\text { Diabetische Nephropathie/ } \\
\text { Glomerulosklerose }\end{array}$} & Diabetes mellitus II & $\begin{array}{l}\text { St. n. Peritonealdialyse } \\
(2 \times \text { Katheter) }\end{array}$ \\
\hline & & & & Rezessive Septikämien & $\begin{array}{l}\text { Diverse zentralnervöse Ka- } \\
\text { thetereinlagen mit Infekten }\end{array}$ \\
\hline & & & & $\begin{array}{l}\text { Vorfußamputation rechts } \\
\text { bei Weichteilinfekt 03/2018 }\end{array}$ & $\begin{array}{l}\text { Fehlende Option für eine } \\
\text { konventionelle Shuntanlage }\end{array}$ \\
\hline & & & & Mangelernährung & - \\
\hline & & & & $\begin{array}{l}\text { Hypertensive und koronare } \\
\text { Herzkrankheit }\end{array}$ & - \\
\hline \multirow[t]{2}{*}{3} & \multirow[t]{2}{*}{ Männlich } & \multirow[t]{2}{*}{66} & \multirow{2}{*}{$\begin{array}{l}\text { Einzelniere nach Nephrektomie rechts } \\
\text { Fokal segmentale Glomerulosklerose, } \\
\text { Ileumconduit aufgrund multipler Infekte } \\
\text { bei Ureterokutaneostomie links }\end{array}$} & \multirow{2}{*}{$\begin{array}{l}\text { Sensomotorische } \\
\text { Tetraparese nach } \\
\text { HWK-Luxationsfixation } \\
1976\end{array}$} & Limitierte Gefäßoptionen \\
\hline & & & & & $\begin{array}{l}\text { Erwartete schlechte Ent- } \\
\text { wicklung einer Fistel bei } \\
\text { Tetraparese }\end{array}$ \\
\hline \multirow[t]{4}{*}{4} & \multirow[t]{4}{*}{ Weiblich } & \multirow[t]{4}{*}{36} & \multirow{4}{*}{$\begin{array}{l}\text { Familiäre Nephropathie mit segmentaler } \\
\text { Glomerulosklerose und chronisch } \\
\text { interstitieller Entzündung }\end{array}$} & $\begin{array}{l}\text { Schwere pulmonal arteriel- } \\
\text { le Hypertonie (Klasse IV) }\end{array}$ & \multirow[t]{4}{*}{$\begin{array}{l}\text { Kardiopulmonale } \\
\text { Limitierung }\end{array}$} \\
\hline & & & & $\begin{array}{l}\text { Adipositas per magna } \\
(\mathrm{BMI}>46)\end{array}$ & \\
\hline & & & & Diabetes mellitus II & \\
\hline & & & & Schlafapnoesyndrom & \\
\hline
\end{tabular}

Subventil musste technisch angepasst werden, damit das Hauptventil sicher und ohne Gefahr einer Luftaspiration gewechselt werden kann.

Wichtig sind eine gute Schulung des Pflegepersonals, eine gute Dokumentation der Vorgehensweise und eine kontinuierliche Begleitung, damit die notwendige Sicherheit im Umgang mit dem Port erreicht wird. Zentral sind das sichere Konnektieren, Dekonnektieren und Aufbringen der Schutzkappe.

Eine im Vorfeld nicht erkannte Schwierigkeit ist der Umgang mit den nachwachsenden Haaren um den Port, welche beim Anschluss des Adapters und des Deckels nicht in das System eindringen dürfen. Solche Schwierigkeiten werden in den regelmäßigen Sitzungen mit dem Entwicklungsteam besprochen und das Handling kontinuierlich optimiert. In Zusammenarbeit mit der für die Portweiterentwicklung verant- wortlichen Firma Diavantis werden die Erfahrungen analysiert, Korrekturen am System umgesetzt und notwendige Änderungen in der Praxis laufend in der Gebrauchsanleitung geändert.

Weltweit arbeiten Forschungsgruppen an diversen Konzepten mit dem Ziel, die Verlässlichkeit und Langlebigkeit von Gefäßzugängen zu verbessern, um die Komplikationsraten und damit die Folgekosten bedingt durch Revisionen zu senken. Wir gehen davon aus, dass sich der initial höhere Aufwand bei der BAPImplantation über die Zeit durch eine geringere Komplikationsrate relativiert. Die Einfachheit der Konnektion zum Port und die bisher festgestellte niedrige Morbidität sind Anreize genug, die klinische Evaluation des Systems voranzutreiben. Innovationen sind nie gratis zu haben und vergesellschaftet mit neuen und teilweise unbekannten Problemen. Das Risiko einer Meningitis ohne Du- raverletzung ist wohl vernachlässigbar. Wie hoch das Risiko einer Osteomyelitis wegen des durch den Knochen ziehenden Katheters ist, bleibt offen. Die an unserer Institution durchgeführte Untersuchung mit 203 Hörgeräteimplantaten zeigen aber, dass die lokale Infektionsrate für retroaurikulär implantierte Fremdkörper sehr tief ist [4].

\section{》) Der Katheter kann ohne Reoperation über einen Seldinger-Draht gewechselt werden}

Der Katheter kann, mindestens theoretisch, ohne Reoperation über einen Seldinger-Draht gewechselt werden. Bei den beiden bisher notwendigen Subventilwechseln war der Katheter gut mobilisierbar. Die Entwickler arbei- 


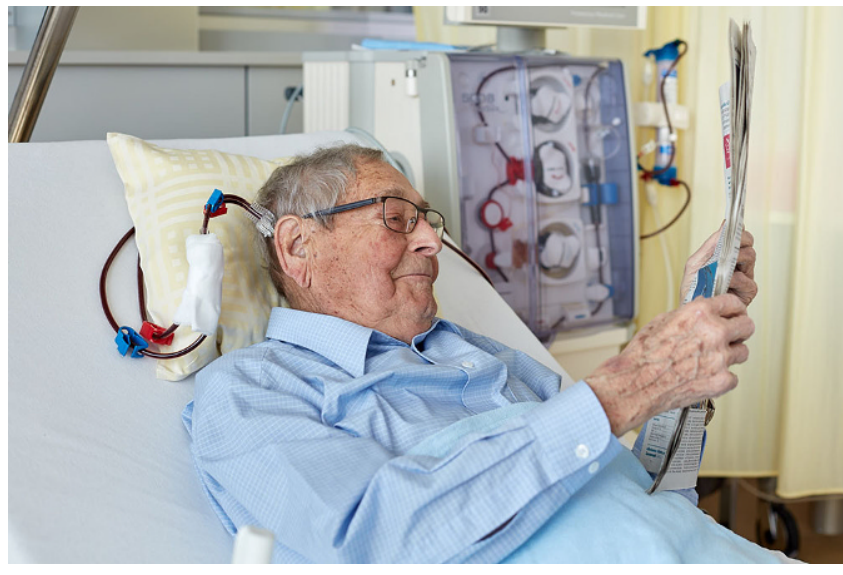

Abb. $11<$ Bequeme und schmerzfreie Hämodialyse über den retroaurikulär fixierten Port (dieser Inhalt ist nicht Teil der Open-Access-Lizenz)

ten an Algorithmen, um bei allfälligen Katheterbakteriämien korrekt zu agieren. Die Szenarien reichen dabei von einem Katheterwechsel, einem temporären Katheterausbau bis hin zu einem kompletten Portausbau.

Eine funktionierende AVF bleibt weiterhin der ideale Gefäßzugang für eine konventionelle, chronische Dialysebehandlung. Kinetische Überlegungen lassen allerdings vermuten, dass mit täglichen HD-Behandlungen oder längeren Behandlungszeiten über Nacht der Outcome verbessert werden kann. AVF kommen bei täglichen Punktionen mit $2 \mathrm{Na}$ deln an ihre Grenzen (und die nächtliche HD im Schlaf mit liegenden Nadeln ist alles andere als ideal) [11]. Mit den sich in Entwicklung befindlichen transportablen künstlichen Nieren wird es mit diesem System möglich sein über längere Zeit und von Laien zu Hause die Prozedur einer Dialyse mit der nötigen Sicherheit durchzuführen.

Die ersten Resultate dieses neuartigen Zugangssystems geben zur Hoffnung Anlass, dass mit diesem ossär verankerten Port der Komfort für HD-Patienten steigt (- Abb. 11). Die Patienten schätzen es sehr, dass keine schmerzhaften Punktionen mehr notwendig sind, sie beide Arme frei gebrauchen können und sie nach der Dialyse rasch nach Hause gehen können, weil es weder ein Abdrücken des Shunts noch einen aufwendigen Verbandswechsel des Dialysekatheters braucht. Bisher hat kein Patient ein Rauschen während der Dialyse bemerkt, was aufgrund der Nähe zum Gehör denkbar wäre. Mit den sich in Entwicklung befindlichen transportablen künstlichen Nieren wird es mit diesem System möglich sein, über längere Zeit und von Laien zu Hause die Prozedur einer Dialyse mit der nötigen Sicherheit durchzuführen.

Soll die Methode weiterverbreitet werden, braucht es ein intensives Schulungskonzept und zu Beginn eine fachliche Supervision durch Kliniker, welche an der Entwicklung dieses Systems von der ersten Stunde an mitgearbeitet haben.

\section{Schlussfolgerungen}

Mit diesem neuen auf dem mastoidfixierten Portsystem sind bisher 4 Patienten problemlos früh postoperativ dialysiert worden. Bisher traten keine Katheterinfekte auf. Es braucht ein interdisziplinäres Team, das sich die einzelnen Operations-/ Behandlungsschritte in Modulen aneignet, um in der Folge unter Supervision die nötige Expertise zu erlangen. Aus pflegerischer Sicht ist das System einfach zu bedienen und die Patienten schätzen das problemlose Konnektieren und Dekonnektieren zur Dialyse.

Die Klappe mit ihrer Membran und die Möglichkeit im Subventil das System zu schließen, geben optimale Sicherheit in der Handhabung dieses Dialysezugangs. Sollten in absehbarer Zukunft transportable künstliche Nieren auf den Markt kommen, wäre dieses System eine Option, um einfach, langdauernd und bequem zu dialysieren.

\section{Fazit für die Praxis}

- Dieser retroaurikulär fixierte Port ist eine innovative Option für HDPatienten, welche keine Vorausset- zungen für eine AV-Fistel haben oder aus anderen Gründen auf einen tunnelierten Dauerkatheter angewiesen sind.

- Die Implantation dieses ausgefeilten Systems braucht viele technische Fertigkeiten und ein interdisziplinäres Team. Es hat den Vorteil, dass man den Adapter zu den Dialyseschläuchen einfach konnektieren und dekonnektieren kann und es wegen des eingebauten Ventils große Sicherheit bietet.

- Es braucht in Zukunft eine intensive Schulung von Behandlungsteams und weitere Evaluationen, damit dieser innovative Port die nötige Verbreitung finden kann.

\section{Korrespondenzadresse

PD Dr. med. et MME
M. K. Widmer
Universitätsklinik für Herz-
und Gefässchirurgie,
Inselspital, Universität Bern
3010 Bern, Schweiz
matthias.widmer@insel.ch

Danksagung. Die Autoren danken Herrn Gianni Pauciello von der Universitätsklinik für HNO für das Bildmaterial, Dr. Thomas Wyss von der Universitätsklinik für Herz- und Gefässchirurgie für die kritische Durchsicht des Manuskriptes und der Kommission für Technik und Innovation (KTI), Schweiz, für die finanzielle Unterstützung.

\section{Einhaltung ethischer Richtlinien}

Interessenkonflikt. M.K. Widmer, A. Arnold, D.E. Uehlinger und $M$. Caversaccio waren zusammen mit der Firma Cendres-Métaux SA (Nachfolge-Firma Diavantis) in der Produkteentwicklung involviert und haben einen Beraterstatus. U. Dietrich gibt an, dass kein Interessenkonflikt besteht.

Wir haben dievon derEthik-Kommission Bern bewilligte Studie an Menschen gemacht, die ihre Zustimmung zur Studie gegeben haben.

Open Access Dieser Artikel wird unter der Creative Commons Namensnennung 4.0 International Lizenz (http://creativecommons.org/licenses/by/4.0/deed. de) veröffentlicht, welche die Nutzung, Vervielfältigung, Bearbeitung, Verbreitung und Wiedergabe in jeglichem Medium und Format erlaubt, sofern Sie den/die ursprünglichen Autor(en) und die Quelle ordnungsgemäßnennen, einen Linkzur Creative Commons Lizenz beifügen und angeben, ob Änderungen vorgenommen wurden. 


\section{Literatur}

1. Almasri J, Alsawas M, Mainou M et al (2016) Outcomes of vascular access for hemodialysis: A systematic review and meta-analysis. J Vasc Surg 64:236-243

2. Arnold A, Kruse A, Wiedmer S, Widmer M, Guignard J, Schütz D, Guenat J-M, Bachtler M, Caversaccio M, Uehlinger DE, Frey FJHRSC (2015) Neuartiger knochenverankerter Hämodialysezugang. Ther Umsch 72:519-524

3. Bray BD, Boyd J, Daly C et al (2012) Vascular access type and risk of mortality in a national prospective cohort of haemodialysis patients. QJM 105:1097-1103

4. Candreia C, Birrer R, Fistarol S et al (2016) Predisposing factors for adverse skin reactions with percutaneous bone anchored hearing devices implanted with skin reduction techniques. Eur Arch Otorhinolaryngol 273:4185-4192

5. Guignard J, Arnold A, Weisstanner C et al (2013) A Bone-thickness map as a guide for Bone-anchored port implantation surgery in the temporal Bone. Materials (Basel) 6:5291-5301

6. Guignard J, Terefe MB, Uehlinger DE, Czerwinska J (2014) Hemodynamics and flow characteristics of a new dialysis port. ASAIO J60:81-89

7. Ishani A, Collins AJ, Herzog CA, Foley RN (2005) Septicemia, access and cardiovascular disease in dialysis patients: The USRDS Wave 2 Study. Kidney Int 68:311-318

8. Klevens RM, Edwards JR, Andrus ML et al (2007) Special report: dialysis surveillance report:national Healthcare safety network (NHSN)-data summary for 2006. Semin Dial 21:24-28

9. Lok CE, Foley R (2013) Vascular access morbidity and mortality: trends of the last decade. Clin J Am Soc Nephrol 8:1213-1219. https://doi.org/10. 2215/CJN.01690213

10. Ravani P, Palmer SC, Oliver MJ et al (2013) Associations between hemodialysis access type and clinical outcomes: a systematic review. J Am Soc Nephrol 24:465-473

11. Rocco MV, Lockridge RS, Beck GJ et al (2011) The effects of frequent nocturnal home hemodialysis: the frequent hemodialysis network nocturnal trial. Kidney Int 80:1080-1091

12. Stieger C, Arnold A, Kruse A et al (2016) Novel bone-anchored vascular access on the mastoid for hemodialysis: concept and preclinical trials. leee Trans Biomed Eng 63:984-990

\section{Verengte Herzkranzgefäße sind auch ohne Stents gut behandelbar}

Um Verengungen von kleinen Herzkranzgefäßen zu behandeln, werden bisher in der Regel Stents eingesetzt. Nun zeigt eine am Universitätsspital Basel durchgeführte Studie eine gute Alternative auf: Mit Medikamenten beschichtete Ballonkatheter sind genauso sicher und effektiv wie Stents.

Schädigungen in kleinen Herzkranzgefäßen mit weniger als drei Millimetern Durchmesser werden bisher üblicherweise mit dem Einsetzen von Stents behandelt. Diese permanenten Gefäßstützen sorgen dafür, dass die verengten Koronargefäße dauerhaft erweitert werden und das Blut wieder ungehindert zirkulieren kann.

Seit einigen Jahren stehen für die Behandlung von solchen Stenosen auch mit Medikamenten beschichtete Ballonkatheter zur Verfügung, die nur vorübergehend in das betroffene Gefäß eingeführt und dann wieder herausgezogen werden. Eine federführend am Universitätsspital Basel (USB) durchgeführte Studie hat nun die beiden Methoden miteinander verglichen.

Es handelt sich um die bisher größte Studie, welche die Wirkung von Medikamentenbeschichteten Ballons (drug-coated balloons, DCB) und Medikamenten-beschichteten Stents (drug-eluting stents, DES) als Therapien bei verengten Herzkranzgefäßen einander gegenübergestellt hat. Zwischen 2012 und 2017 wurden an 14 Spitälern in Deutschland, Österreich und der Schweiz 758 Patientinnen und Patienten in die Studie einbezogen. In randomisierter Weise wurde die eine Hälfte mit Stents und die andere Hälfte mit Ballonkathetern behandelt.

\section{Methode mit Ballonkatheter hat Vorteile}

Die Auswertung der Daten hat gezeigt, dass mit beiden Methoden gleichwertig gute Behandlungsergebnisse erzielt werden. Bei beiden Verfahren war die Rate an schwerwiegenden unerwünschten kardiovaskulären Ereignissen wie Herzinfarkt oder Tod zwölf Monate nach dem Eingriff praktisch gleich tief. Somit lässt sich die Aussage machen, dass Stenosen in kleinen Koronargefäßen mit Ballonkathetern sicher behandelt werden können, so dass der Einsatz von Stents nicht zwingend notwendig ist.

Für Studienleiter Prof. Raban Jeger, Leitender Arzt Kardiologie am USB, hat der Einsatz von Ballonkathetern Vorteile: „Mit dem Verzicht auf Stents kann zum Beispiel die normale Ge- fäßfunktion erhalten werden. Außerdem ist die Behandlungszeit mit Plättchenhemmern kürzer, und es können vor allem keine Stentthrombosen auftreten." Aus diesen Gründen und der nun vorliegenden wissenschaftlichen Evidenz wird das Verfahren mit Ballonkatheter mittlerweile am USB standardmäßig eingesetzt.

Quelle: Jeger RV et al. Drug-coated balloons for small coronary artery disease (BASKET-SMALL 2): an openlabel randomised non-inferiority trial. Lancet. 2018 Aug 28. https://doi.org/10.1016/S01406736(18)31719-7 Universitätsspital Basel 